\title{
BMJ Open Fruit and vegetable intake and risk of type 2 diabetes mellitus: meta-analysis of prospective cohort studies
}

\author{
Min Li, ${ }^{1}$ Yingli Fan, ${ }^{2}$ Xiaowei Zhang, ${ }^{1}$ Wenshang Hou, ${ }^{1}$ Zhenyu Tang ${ }^{1}$
}

To cite: Li M, Fan Y, Zhang $X$, et al. Fruit and vegetable intake and risk of type 2 diabetes mellitus: meta-analysis of prospective cohort studies. BMJ Open 2014;4:e005497.

doi:10.1136/bmjopen-2014005497

- Prepublication history and additional material is available. To view please visit the journal (http://dx.doi.org/ 10.1136/bmjopen-2014005497).

Received 22 April 2014 Revised 11 September 2014 Accepted 15 September 2014

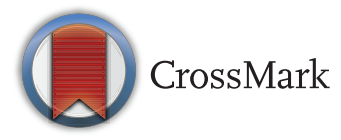

${ }^{1}$ Department of Neurology, The Second Affiliated Hospital of Nanchang University, Nanchang, Jiangxi Province, People's Republic of China

${ }^{2}$ Department of

Cardiovascular, The Second Affiliated Hospital of

Nanchang University, Nanchang, Jiangxi Province, People's Republic of China

Correspondence to

Dr Z Tang;

zytang07016@sina.com

\section{ABSTRACT}

Objective: To clarify and quantify the potential doseresponse association between the intake of fruit and vegetables and risk of type 2 diabetes.

Design: Meta-analysis and systematic review of prospective cohort studies.

Data source: Studies published before February 2014 identified through electronic searches using PubMed and Embase.

\section{Eligibility criteria for selecting studies:}

Prospective cohort studies with relative risks and $95 \%$ Cls for type 2 diabetes according to the intake of fruit, vegetables, or fruit and vegetables.

Results: A total of 10 articles including 13 comparisons with 24013 cases of type 2 diabetes and 434342 participants were included in the metaanalysis. Evidence of curve linear associations was seen between fruit and green leafy vegetables consumption and risk of type 2 diabetes $(p=0.059$ and $\mathrm{p}=0.036$ for non-linearity, respectively). The summary relative risk of type 2 diabetes for an increase of 1 serving fruit consumed/day was $0.93(95 \% \mathrm{Cl} 0.88$ to 0.99 ) without heterogeneity among studies $(p=0.477$, $\mathrm{I}^{2}=0 \%$ ). For vegetables, the combined relative risk of type 2 diabetes for an increase of 1 serving consumed/ day was $0.90(95 \% \mathrm{Cl} 0.80$ to 1.01$)$ with moderate heterogeneity among studies $\left(p=0.002, l^{2}=66.5 \%\right)$. For green leafy vegetables, the summary relative risk of type 2 diabetes for an increase of 0.2 serving consumed/day was $0.87(95 \% \mathrm{Cl} 0.81$ to 0.93$)$ without heterogeneity among studies $\left(p=0.496, l^{2}=0 \%\right)$. The combined estimates showed no significant benefits of increasing the consumption of fruit and vegetables combined.

Conclusions: Higher fruit or green leafy vegetables intake is associated with a significantly reduced risk of type 2 diabetes.

\section{INTRODUCTION}

Type 2 diabetes (T2D) is one of the most common non-communicable diseases which is expected to affect in excess of 439 million adults worldwide by $2030,{ }^{1}$ with serious consequences for healthcare expenditure. ${ }^{2}$ It has been estimated that the global health expenditure on diabetes is at least $\$ 376$

\section{Strengths and limitations of this study}

To the best of our knowledge, this is the largest systematic review and meta-analysis on the intake of fruit and vegetables and risk of type 2 diabetes. We also investigated a dose-response relation between fruit, vegetables, or fruit and vegetables combined consumption and risk of type 2 diabetes.

- The possibility of residual confounding or confounding by unmeasured factors, which cannot be ruled out in any observational study, must be acknowledged. We cannot exclude the possibility of recall bias in the assessments of diet based on the food frequency questionnaires.

- Health expenditure on type 2 diabetes is increasing worldwide.

- Epidemiological studies suggest that the intake of fruit and vegetables is beneficial in delaying or preventing the development of type 2 diabetes, though results from cohort studies are controversial.

- Higher fruit or vegetables, particularly green leafy vegetables intake, is associated with a significantly reduced risk of type 2 diabetes.

- Dose-response analyses indicated a $6 \%$ lower risk of type 2 diabetes per 1 serving/ day increment of fruit intake and a $13 \%$ lower risk of type 2 diabetes per 0.2 serving/day increment of green leafy vegetables intake.

- Further evidence from preferably randomised controlled studies should explore what kind of fruit or green leafy vegetables can reduce the risk of type 2 diabetes.

billion in 2010 and will be $\$ 490$ billion in $2030,{ }^{3}$ which creates a major public health burden. The prevention of T2D is thus clearly an important public health priority. In recent decades, concern has mounted regarding the premature mortality and morbidity associated with T2D, with growing interest in altering risk factors and reversing this global epidemic. Among the known risk factors for T2D, dietary factors have aroused particular attention. Lifestyle intervention trials that include dietary modification have 
been shown to be effective in delaying or preventing the development of T2D. ${ }^{4}$ Although the effect of individual components or interactions between nutrients is still largely unknown, fruit and vegetables intake may explain some of this beneficial effect. ${ }^{5}$

To minimise the risk of dietary factors and reduce the incidence of T2D, the WHO has recommended the public for consuming more than $400 \mathrm{~g}$ or five portions of combined fruit and vegetables per day to prevent T2D. ${ }^{6}$ Nevertheless, in the Japan Public Health Center-based Prospective (JPHC) Study, ${ }^{7}$ after a mean follow-up over 5 years, participants with the intake of fruit and vegetables may not be appreciably associated with the risk of T2D. Vegetables, especially green leafy vegetables (GLV), have been suggested to explain an apparent beneficial effect on T2D. In addition, several meta-analyses of observational studies have found that an increase in the daily intake of GLV could significantly reduce the risk of T2D. ${ }^{589}$ These studies were restricted by heterogeneity with respect to sample size. Additionally, recent studies involving a relationship between the intake of fruit and vegetables and the risk of T2D have been published from

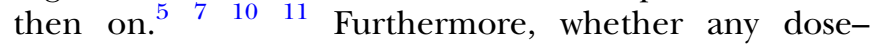
response relation exists between the intake of fruit and vegetables and the risk of T2D is unknown. Therefore, we systematically reviewed and meta-analysed available studies to quantify the associations between dietary intake of fruit and vegetables and incidence of T2D based on identified prospective cohort studies. We pooled risk estimates for the highest versus lowest category of intake to examine the overall association. We also conducted a dose-response analysis for the trend estimation.

\section{METHODS}

\section{Search strategy}

We carried out a systematic search of PubMed (MEDLINE) and Embase through February 2014 for prospective cohort studies examining the association between the intake of fruit and vegetables and risk of T2D. The following key words were used in our search strategies: ('fruits' OR 'vegetables' OR 'citrus') AND ('Type 2 diabetes' OR 'non-insulin-dependent diabetes mellitus' OR 'Diabetes Mellitus, Type 2' OR 'NIDDM' OR 'prediabetes' OR 'impaired glucose tolerance' OR 'impaired fasting glucose' OR 'glucose' OR 'hyperglycaemia' OR 'insulin') AND ('Follow-up studies' OR 'prospective studies' OR 'cohort studies' OR 'longitudinal studies'). We restricted the search to human studies. No language restrictions were imposed. In addition, we scrutinised possible eligible references from relevant original papers and review articles to identify potential publications. We followed standard criteria for the performing and reporting of the meta-analyses of observational studies. ${ }^{12}$

\section{Study selection}

Citations selected from the initial search were subsequently screened for eligibility. Studies were included in this meta-analysis if they satisfied the following criteria: (1) original studies (eg, not review articles, meeting abstracts, editorials or commentaries); (2) prospective design (eg, not cross-sectional design, case-control design); (3) the exposure of interest was the intake of fruits, vegetables, or fruit and vegetables combined; (4) the outcome was T2D and (5) reported multivariateadjusted risk estimates for the association between the fruit, vegetables, or fruit and vegetables combined, assessed as dietary intake, and T2D. Additionally, we excluded animal studies and letters without sufficient data. If data were reported more than once, we included the study with the longest follow-up time.

\section{Validity assessment}

Two authors (ML and YF) independently assessed all studies for quality using a modified scoring system, which allowed a total score from 0 to 6 points ( 6 reflecting the highest quality) on the basis of MOOSE (Meta-analysis Of Observational Studies in Epidemiology), ${ }^{12}$ QUATSO (quality assessment tool for systematic reviews of observational studies), ${ }^{13}$ and STROBE (Strengthening the Reporting of Observational Studies in Epidemiology). ${ }^{14}$ The system was created to account for study eligibility (1 point for appropriate inclusion and exclusion criteria), outcome (1 point if diagnosis of T2D was based on accepted clinical criteria, and not solely based on selfreport), exposure (1 point if fruit and vegetables consumption was assessed with a validated tool, and 1 point if fruit and vegetables consumption was appropriately categorised), statistical analysis (1 point was given if adjustment included a few variables such as age, sex, body mass index and family history of T2D, these being proven risk factors for T2D). Another point was given if any other factors were adjusted (such as alcohol, education and physical activity). ${ }^{9}$

\section{Data extraction}

Data extractions were carried out independently by two other authors ( $\mathrm{XZ}$ and $\mathrm{WH}$ ) using standard electronic sheets and cross-checks to reach a consensus. For each study, the following information was abstracted: name of the first author, publication year, study population, geographical location, sex, age range, sample size (number of T2D cases, number of non-T2D cases and number of participants), duration of follow-up, methods used to assess fruit and vegetables intake and ascertain T2D cases, highest and lowest intake of fruit and vegetables, and covariates adjusted for in the multivariable model. Study quality was evaluated by using the modified scoring system. All data were extracted from the published papers. If necessary, the primary authors were contacted to retrieve further information. For two studies that expressed data separately for men and women, ${ }^{715}$ including one study that included data from multiple cohorts, ${ }^{11}$ we considered the analysis for each sex or cohort as an independent comparison and extracted data separately. 


\section{Statistical analysis}

Within each study, we used multivariate-adjusted outcome data (expressed as relative risks and 95\% CIs) for risk estimates. For the present analyses, we assumed HRs to be a valid approximation of relative risks and converted these values in every study by taking their natural logarithms and calculating SEs and corresponding 95\% CIs. Relative risks and their SEs were pooled with the DerSimonian and Laird random effects model, which takes into account both within-study and betweenstudy variabilities. ${ }^{16}$ When some studies included in our meta-analysis used different measurement units (eg, grams per day or portions per day or servings per day), ${ }^{5} 1015$ we standardised fruit and vegetables intake into servings per day using a standard portion size of 106 g. ${ }^{17}$ As different studies might use different exposure categories (thirds, quarters or fifths), ${ }^{711} 15$ we used the study-specific relative risk for the highest versus lowest category of fruit, vegetables, or fruit and vegetables intake for the meta-analysis. For the dose-response analysis, the generalised least square for trend estimation method described by Greenland and Longnecker ${ }^{18}$ and Orsini et $a l^{1920}$ was used to calculate study-specific slopes (linear trends) and 95\% CIs. The method requires the distributions of cases and person years for exposure categories, and median/mean of fruit, vegetables, or fruit and vegetables intake levels for each comparison group. We assigned the midpoint of the upper and lower boundaries of each comparison group to determine mean fruit, vegetables, or fruit and vegetables intake levels if the median or mean intake was not provided. When the highest category was open ended, we assumed that the average of the category was set at 1.5 times the lower boundary. Additionally, we first created restricted cubic splines with 4 knots at 5th, 35th, 65th and 95th centiles of the distribution. ${ }^{21} \mathrm{~A} p$ value for non-linearity was calculated by testing the null hypothesis that the coefficient of the fractional polynomials component is equal to zero. Heterogeneity among studies was evaluated using the $\chi^{2}$ test based on Cochran's $Q$ test and $\mathrm{I}^{2}$ statistic at the $\mathrm{p}<0.10$ level of significance, ${ }^{16}$ and quantification of heterogeneity was made by the $\mathrm{I}^{2}$ metric, which describes the percentage of total variation in point estimates that is due to heterogeneity rather than chance. ${ }^{22}$ We considered low, moderate and high degrees of heterogeneity to be $\mathrm{I}^{2}$ values of $25 \%, 50 \%$ and $75 \%$, respectively. To explore possible explanations for heterogeneity and to test the robustness of the association, we conducted subgroup analyses based on the location (Asia vs Non-Asia), the quality of the study (high quality $(\geq 4)$ vs lower quality $(<4)$ ), length of follow-up ( $\geq 10$ years vs $<10$ years), sex (male and female included vs female only vs male only), fractions of intake (thirds, quarters or fifths), number of participants $(\geq 50000$ vs $<50000)$, and number of cases $(\geq 1000$ vs $<1000)$. We also performed the Begg rank correlation test and Egger's regression test to visualise a possible asymmetry. ${ }^{23-25}$ All the statistical analyses were performed in Stata V.12 (StataCorp, College Station, Texas, USA). A threshold of $\mathrm{p}<0.1$ was used to decide whether heterogeneity or publication bias was present. ${ }^{24}$ In other ways, $p$ values were two-sided and $p<0.05$ was considered statistically significant.

\section{RESULTS}

\section{Literature search}

Figure 1 shows the results of literature research and selection. We identified 308 articles from PubMed and 365 articles from Embase. After exclusion of duplicate records and studies that did not fulfil our inclusion criteria, 27 articles remained, and we further evaluated the full texts of these 27 publications. Of these, we excluded 17 studies as follows. Five articles were excluded owing to lack of sufficient data for estimation of relative risks. ${ }^{26-30}$ Five articles were excluded because no original data could be extracted (review, type 1 diabetes or cross-sectional studies)..$^{31-35}$ Another four articles were excluded because we deemed them to be irrelevant. ${ }^{36-39}$ We also excluded three articles because they did not give enough details on fruit, vegetables, or fruit and vegetables intake to warrant inclusion within the meta-analysis. ${ }^{40-42}$ Finally, 10 articles met the inclusion criteria and were included in the meta-analysis. ${ }^{5} 71011 \quad 15$ 43-47 Among these articles, two studies provided information on males and females separately; ${ }^{71}$ the article by Cooper et al was divided into two studies (study a:2012 and study b:2012); and another paper reported data from two independent cohorts. ${ }^{15}$ Thus, our meta-analysis included 13 comparisons.

\section{Study characteristics}

Online supplementary tables A and B (appendix 1) show the characteristics extracted from the included studies; all 10 articles were prospective cohort designs

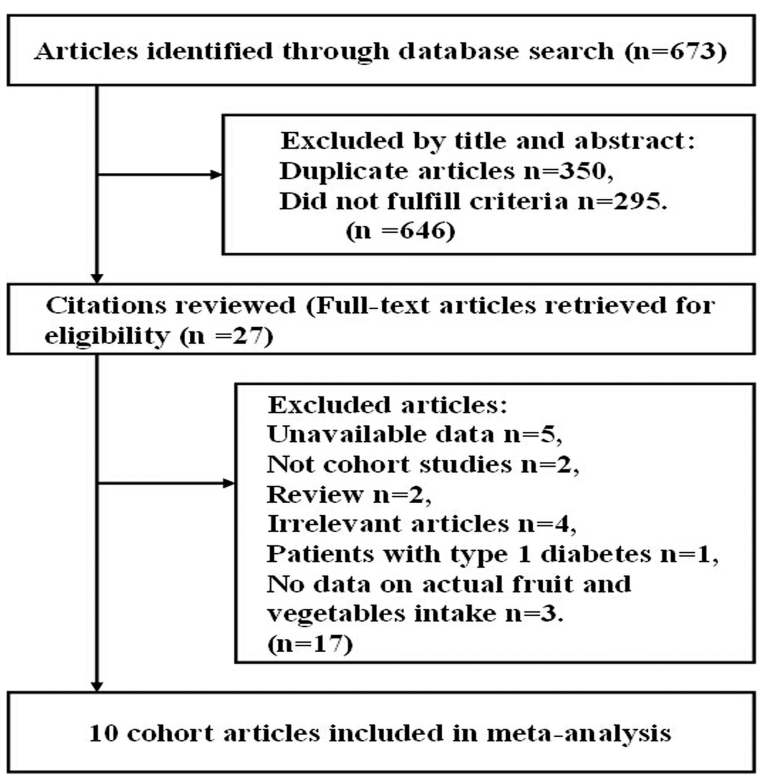

Figure 1 Process of literature search and study selection. 
and participants who were free of self-reported diabetes

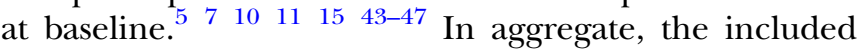
studies consisted of 434342 participants. Among the participants, we documented 24013 cases of T2D which occurred during follow-up periods ranging from 4.6 to 23 years (median of 11 years). Five cohorts were conducted primarily in the USA, ${ }^{11} 15434447$ two in Asian countries (China and Japan) ${ }^{7} 46$ and three in European countries. ${ }^{5} 1045$ The number of participants ranged from 3704 in the European Prospective Investigation into Cancer and Nutrition (EPIC) Norfolk study by Cooper et $a l^{10}$ to 91246 in the Nurses' Health Study II by Muraki et al. ${ }^{11}$ Five studies included both male and females, ${ }^{5} \quad 7 \quad 10 \quad 15 \quad 45$ four studies included only females. ${ }^{43} 444647$ One article by Muraki et $^{4 l^{11}}$ reported two independent cohorts; one cohort included only females, and another included only males. The age of participants ranged from 24 to 79 years. Six papers provided information on fruit and vegetables intake separately and combined, ${ }^{5} 710434447$ two papers provided information on fruit and vegetables intake separately, ${ }^{45} 46$ one paper provided only the combined data, ${ }^{15}$ and another paper provided separate data on fruit. ${ }^{11}$ Five papers also included separate data on the intake of GLV. ${ }^{5} 74-46$ In most papers, the intake of fruit and vegetables was divided into fifths. ${ }^{11}{ }^{43-47}$ All studies provided adjusted risk estimates; results of study quality assessment (score 0-6) showed that most studies yielded a score of 3 or below (low quality).

\section{Fruit intake and risk of T2D}

Eleven comparisons from nine studies reported an association between fruit intake and risk of T2D, with 22995 T2D outcomes and 424677 participants. Overall, fruit intake was inversely associated with risk (relative risk $0.93,95 \%$ CI 0.88 to 0.99 ; figure 2 ). We saw no heterogeneity among studies $\left(\mathrm{p}=0.477, \mathrm{I}^{2}=0 \%\right)$. Additionally, no evidence of substantial publication bias was observed from the Begg $(p=0.533)$ and Egger regression tests ( $\mathrm{p}=0.849$; see online supplementary table B in appendix 1). Among 11 comparisons, 7 were eligible for the dose-response analysis of fruit intake and risk of T2D. Using a restricted cubic splines model, we found a mild curvilinear association ( $\mathrm{p}=0.059$ for non-linearity, figure $3)$. Dose-response analysis indicated that a 1 serving/ day increment of fruit intake was associated with a $6 \%$ lower risk of T2D (relative risk $0.94,95 \%$ CI 0.89 to 1.00 , $\mathrm{I}^{2}=0 \%$; see online supplementary figure $\mathrm{A}$ in appendix 2).

\section{Vegetables intake and risk of T2D}

Eight studies exported an association between vegetables intake and risk of T2D, with 20933 T2D outcomes and 290927 participants. Using a random effects model summarising all nine comparisons, we found no association between vegetables intake and risk (relative risk 0.90, $95 \%$ CI 0.80 to 1.01 ; figure 4 ). There was moderate study heterogeneity $\left(\mathrm{p}=0.002, \mathrm{I}^{2}=66.5 \%\right)$. However, no

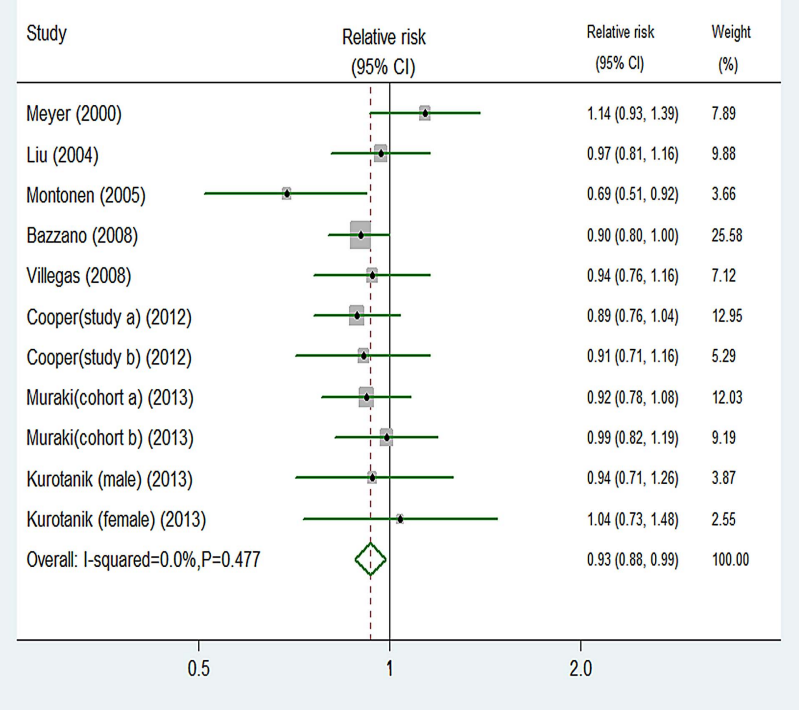

Figure 2 Random effects analysis of fully adjusted studies for highest versus lowest intake of fruit and risk of type 2 diabetes.

evidence of substantial publication bias was observed from the Begg $(p=0.602)$ and Egger regression tests ( $p=0.176$; see online supplementary table $B$ in appendix $1)$. Among nine comparisons, five were eligible for the trend estimation. Dose-response analysis found no association with risk of T2D per 1 serving/day increment of vegetables intake (relative risk $0.98,95 \%$ CI 0.89 to 1.08 , $\mathrm{I}^{2}=45.8 \%$; see online supplementary figure $\mathrm{B}$ in appendix 2). No publication bias was observed $(p=0.117)$. We found no evidence of a curve linear association between

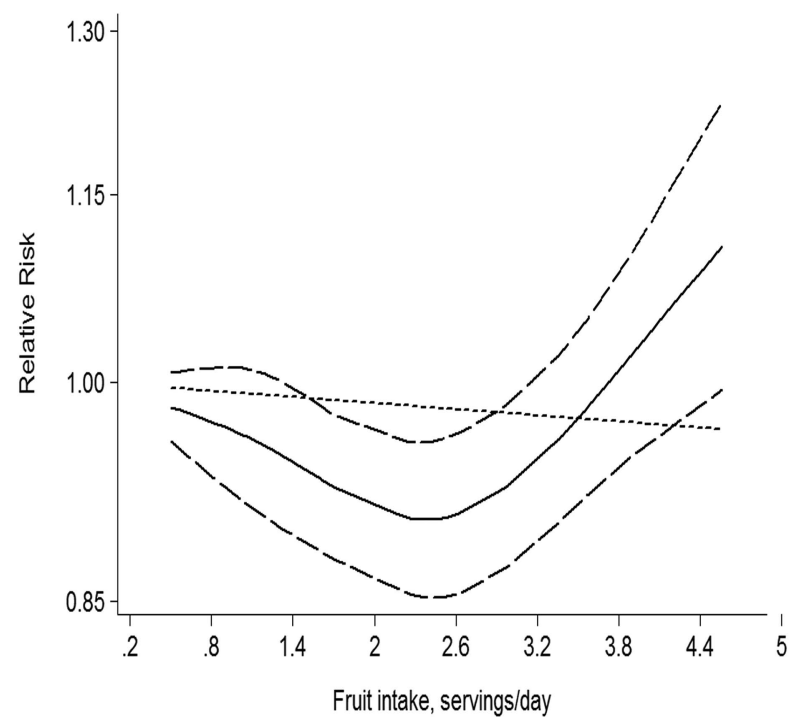

Figure 3 Dose-response analyses of fruit intake and risk of type 2 diabetes. 


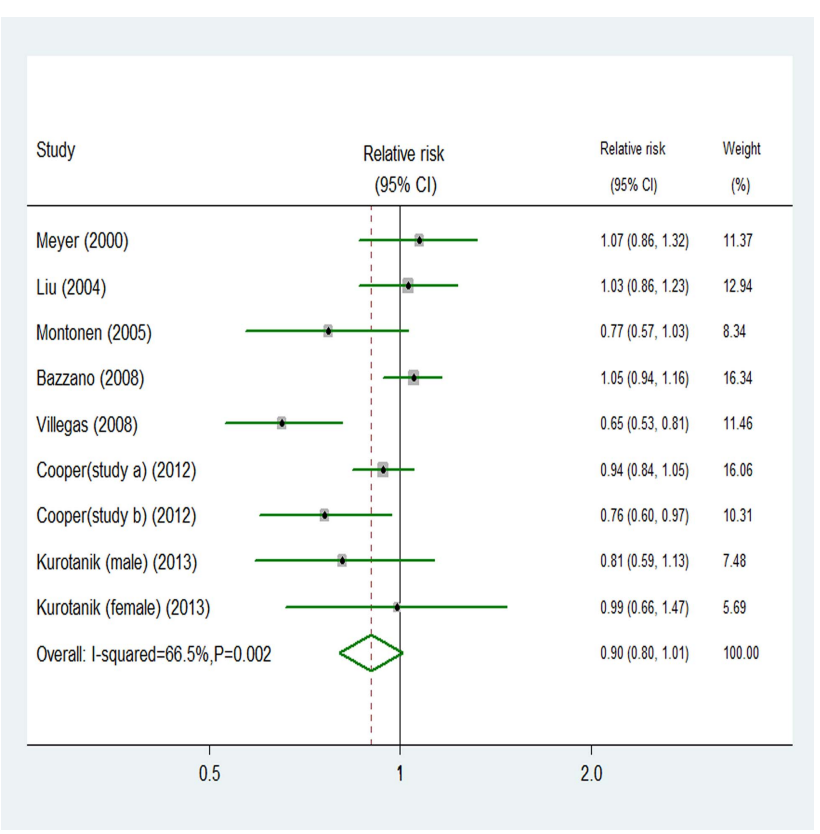

Figure 4 Random effects analysis of fully adjusted studies for highest versus lowest intake of vegetables and risk of type 2 diabetes.

vegetables intake and risk ( $\mathrm{p}=0.671$ for non-linearity, see online supplementary figure $\mathrm{C}$ in appendix 2).

\section{Fruit and vegetables intake and risk of $\mathrm{T} 2 \mathrm{D}$}

Information on fruit and vegetables intake and T2D were available in nine comparisons from seven prospective studies, totalling 20672 T2D outcomes and 232097 participants. Overall, fruit and vegetables intake was not associated with risk (relative risk $0.94,95 \%$ CI 0.86 to 1.03; see online supplementary figure $\mathrm{D}$ in appendix 2). We saw no heterogeneity among studies $(\mathrm{p}=0.141$, $\left.\mathrm{I}^{2}=34.6 \%\right)$. Additionally, no evidence of substantial publication bias was observed from the Begg $(p=0.348)$ and Egger regression tests $(\mathrm{p}=0.609$; see online supplementary table $\mathrm{B}$ in appendix 1). Among nine comparisons, six were eligible for the dose-response analysis of fruit and vegetables intake and risk of T2D. We did not find a significant curvilinear association $(p=0.456$ for nonlinearity, see online supplementary figure $\mathrm{E}$ in appendix 2). Dose-response analysis showed no association between risk of T2D and a 1 serving/day increment of fruit and vegetable intake (relative risk 0.96, 95\% CI 0.86 to $1.07, \mathrm{I}^{2}=47.6 \%$; see online supplementary figure F (appendix 2)).

\section{GLV intake and risk of T2D}

Seven comparisons from six studies reported an association between GLV intake and risk of T2D, with 19139 T2D outcomes and 251235 participants. Overall, GLV intake was inversely associated with risk (relative risk $0.87,95 \%$ CI 0.81 to 0.93 ; figure 5 ). No significant heterogeneity was detected among studies $(\mathrm{p}=0.496$, $\mathrm{I}^{2}=0 \%$ ). Additionally, we did not observe evidence of

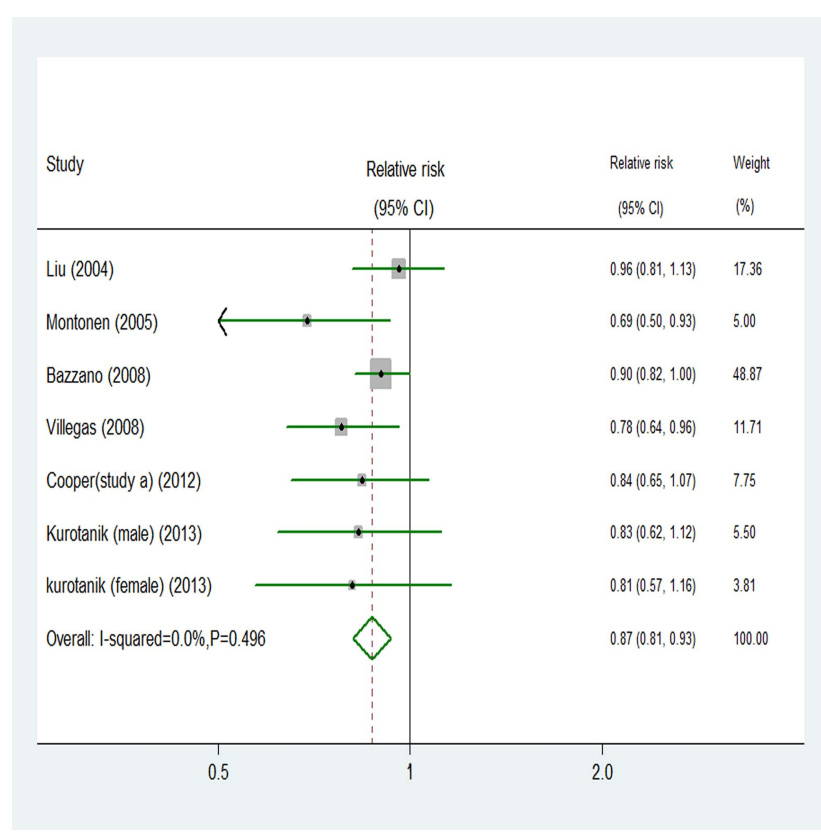

Figure 5 Random effects analysis of fully adjusted studies for highest versus lowest intake of green leafy vegetables and risk of type 2 diabetes.

substantial publication bias (Begg and Egger regression tests, $\mathrm{p}=0.133$ and $\mathrm{p}=0.101$, respectively; see online supplementary table B in appendix 1). Among seven comparisons, only three comparisons were eligible for the trend estimation. Using a restricted cubic splines model, we found a significant curvilinear association $(p=0.036$ for non-linearity, figure 6). Dose-response analysis indicated that a 0.2 serving/day increment of GLV intake was associated with a $13 \%$ lower risk of T2D (relative risk $0.87,95 \%$ CI 0.76 to $0.99, \quad \mathrm{I}^{2}=20.9 \%$; see online

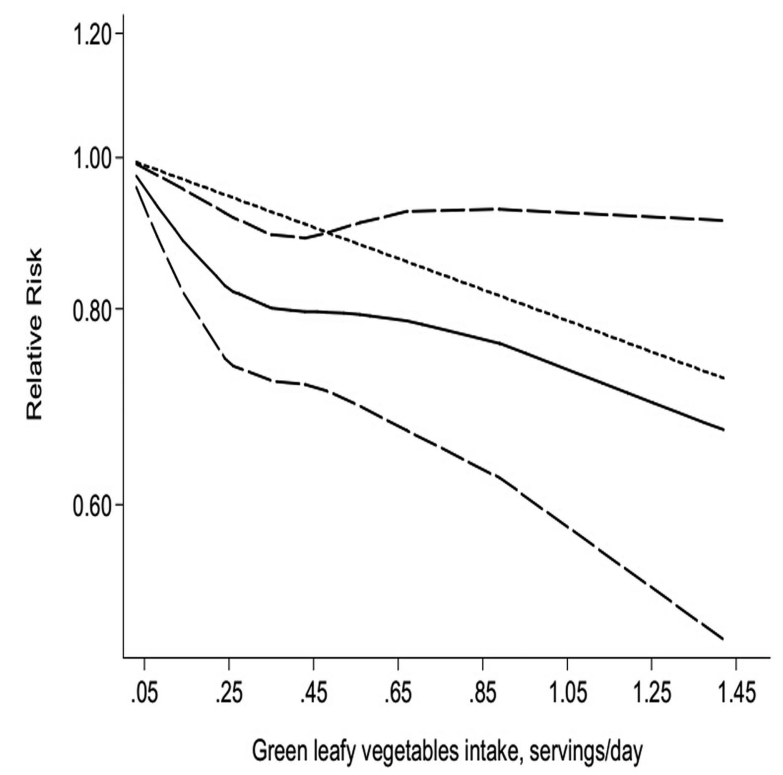

Figure 6 Dose-response analyses of green leafy vegetables intake and risk of type 2 diabetes. 
supplementary figure $\mathrm{G}$ in appendix 2). No publication bias was observed $(\mathrm{p}=0.282)$.

\section{Subgroup analyses}

To examine the stability of the primary results, we carried out subgroup analyses (table 1). The association between fruit, vegetables, or fruit and vegetables intake and risk of T2D was similar in subgroup analyses, which were separately defined; study quality, length of follow-up, sex, location, number of cases or participants, and whether the different ways in which authors had grouped intake (thirds, quarters or fifths) affected the results. The summary estimates of relative risks from each category were pooled (see online supplementary table $\mathrm{B}$ in appendix 1). We paid close attention to the highest versus lowest category. Almost all subgroups that analysed intake of GLV showed a benefit of consuming greater quantities (figure 5). Online supplementary table B in appendix 1 also showed significant reductions in risk of T2D events for consumption of fruit, vegetables, or fruit and vegetables combined.

\section{DISCUSSION}

In this meta-analysis, dietary intake of fruit, vegetables, and GLV, but not fruit and vegetables combined, were associated with a lower risk of T2D. Dose-response analyses indicated a $6 \%$ lower risk of T2D per 1 serving/day increment of fruit intake and a 13\% lower risk of T2D per 0.2 serving/day increment of GLV intake, but no significant trend for vegetables or fruit and vegetables combined.

\section{Results in relation to other studies}

Over the past decades, extensive prospective studies have reported the association of fruit, vegetables, or fruit and vegetables combined with T2D risk. ${ }^{5} \quad 1011 \quad 1543-47$ However, the role of dietary factors in T2D is still controversial. Some of the studies failed to find an association between fruit intake or fruit and vegetables combined and risk of T2D. ${ }^{843}$ Bazzano et $a l^{47}$ analysed data from 11 different US states with 18 years of follow-up. They found that consumption of fruit was associated with a lower risk of diabetes, but no significant association for total fruit and vegetable consumption. Similar to previous analysis in the Nurses' Health Study, the results from three prospective longitudinal cohort studies also supported an inverse association between fruit intake and risk of T2D. ${ }^{11}$ However, these studies have a potential for bias due to measurement error. In addition, two cohort studies have suggested an inverse association between total fruit and vegetables consumption and risk of T2D. ${ }^{1015}$

A few large cohort studies have found an inverse association between vegetables consumption, especially GLV, and risk of T2D. ${ }^{10}{ }^{44-46}$ These findings all agreed with two meta-analyses. ${ }^{5}$ However, another systematic review based on five cohort studies suggested that there was no protective association between vegetables intake and T2D. ${ }^{8}$

Several plausible biological mechanisms have been proposed to explain the aforementioned association. Fruit and vegetables are rich in fibre ${ }^{48}$ which has been shown to improve insulin sensitivity and insulin secretion to overcome insulin resistance. ${ }^{49}$ However, meta-analyses showed that fruit and vegetable fibre is inconsistently associated with the risk of T2D. ${ }^{50}$ On the other hand, it may contribute to a decreased incidence of T2D through their low-energy density and glycaemic load, and high micronutrient content. ${ }^{51}$ In particular, GLV are rich in bioactive phytochemicals (such as vitamin $\mathrm{C}$ and carotenoids), which are known for their antioxidant properties. ${ }^{52-54}$ Antioxidants in fruit and vegetables have been hypothesised to improve insulin sensitivity and protect against diabetes in several supplementation trials. ${ }^{55}$ In addition, it might also reduce the risk of T2D due to the supply of magnesium $(\mathrm{Mg})$; a recent meta-analysis detected $\mathrm{Mg}$ intake to be inversely associated with the risk of T2D. ${ }^{57}$ Taking this evidence into consideration, it appears that the beneficial effects of vegetables, particularly GLV consumption on the risk of T2D, can be mainly explained by antioxidant vitamins and magnesium. The inverse association may be also mediated through weight gain or obesity, which is an established risk factor for T2D. Fruits are low in energy, which would promote the feeling of fullness and prevent overconsumption of energy-dense foods, and result in weight loss. ${ }^{54}$ Further investigation is warranted to understand the mechanisms involved in the proposed relation between fruit, vegetables or GLV and risk of T2D.

\section{Exploration of heterogeneity}

Heterogeneity between studies was found, which did not alter much in the subgroup analyses. There are differences in the types of vegetable consumed between Asian (such as China) and Non-Asian populations. Therefore, within the subgroup analysis, we examined location as a possible source of heterogeneity. As traditional Chinese diets are high in vegetables (such as GLV and cruciferous vegetables), unsurprisingly, vegetables (including GLV) intake was greater in China than in the USA or Europe. We also examined study quality, length of follow-up, sex, number of cases or participants, and whether the different ways in which authors had grouped intake (thirds, quarters or fifths) as possible sources of heterogeneity; these did not show any significant heterogeneity between studies. Although the subgroup analysis could not explain the level of heterogeneity in interpreting the results, several differences between the studies are worth discussing.

Assessment methods of fruit, vegetables, or fruit and vegetables combined consumption differed between the studies. Most epidemiological studies used the food frequency questionnaires (FFQs) to assess the quantity of fruit, vegetables, or fruit and vegetables combined intake. $^{7} \quad 101143444647$ FFQs are less suitable for the 
Table 1 Subgroup analyses to investigate differences between studies included in meta-analysis (highest vs lowest category)

\begin{tabular}{|c|c|c|c|c|c|c|c|c|c|c|c|c|}
\hline \multirow[b]{2}{*}{ Variables } & \multicolumn{3}{|c|}{ Fruit only } & \multicolumn{3}{|c|}{ Vegetables only } & \multicolumn{3}{|c|}{ Fruit and vegetables } & \multicolumn{3}{|c|}{ Green leafy vegetables } \\
\hline & $\mathbf{N}$ & Pooled RR (95\% Cl) & p Value & $\mathbf{N}$ & Pooled RR (95\% Cl) & p Value & $\overline{\mathbf{N}}$ & Pooled RR (95\% Cl) & p Value & $\mathbf{N}$ & Pooled RR (95\% Cl) & p Value \\
\hline \multicolumn{13}{|l|}{ Location } \\
\hline Non-Asia & 8 & $0.93(0.87$ to 1.00$)$ & 0.049 & 6 & $0.96(0.87$ to 1.06$)$ & 0.397 & 7 & $0.94(0.84$ to 1.04$)$ & 0.223 & 4 & 0.89 (0.81 to 0.97$)$ & 0.012 \\
\hline Asia & 3 & 0.96 (0.82 to 1.12$)$ & 0.584 & 3 & 0.77 (0.60 to 0.98$)$ & 0.032 & 2 & 0.97 (0.75 to 1.25$)$ & 0.827 & 3 & 0.80 (0.69 to 0.93$)$ & 0.004 \\
\hline \multicolumn{13}{|l|}{ Quality } \\
\hline High $(\geq 4)$ & 4 & $0.92(0.86$ to 1.00$)$ & 0.045 & 2 & 0.83 (0.52 to 1.33$)$ & 0.448 & 1 & $1.04(0.87$ to 1.25$)$ & 0.671 & 2 & $0.86(0.76$ to 0.98$)$ & 0.024 \\
\hline Low $(<4)$ & 7 & 0.94 (0.85 to 1.04$)$ & 0.240 & 7 & 0.93 (0.84 to 1.02$)$ & 0.109 & 8 & 0.93 (0.84 to 1.02$)$ & 0.138 & 5 & $0.86(0.77$ to 0.97$)$ & 0.010 \\
\hline \multicolumn{13}{|c|}{ Duration of follow-up (years) } \\
\hline$\geq 10$ & 5 & $0.90(0.83$ to 0.97$)$ & 0.006 & 4 & $0.91(0.79$ to 1.05$)$ & 0.190 & 5 & 0.89 (0.77 to 1.02$)$ & 0.098 & 3 & 0.85 (0.75 to 0.97$)$ & 0.014 \\
\hline$<10$ & 6 & 0.98 (0.90 to 1.07$)$ & 0.654 & 5 & $0.89(0.72$ to 1.10$)$ & 0.296 & 4 & $1.03(0.91$ to 1.16$)$ & 0.674 & 4 & 0.87 (0.78 to 0.97$)$ & 0.013 \\
\hline \multicolumn{13}{|c|}{ (1) } \\
\hline $\mathrm{M}$ and $\mathrm{F}$ & 5 & $0.88(0.79$ to 0.98$)$ & 0.022 & 5 & 0.89 (0.81 to 0.97$)$ & 0.010 & 6 & 0.87 (0.77 to 0.98$)$ & 0.026 & 4 & 0.80 (0.69 to 0.92$)$ & 0.002 \\
\hline F only & 5 & 0.95 (0.88 to 1.02$)$ & 0.168 & 4 & $0.94(0.77$ to 1.15$)$ & 0.544 & 3 & $1.02(0.94$ to 1.11$)$ & 0.610 & 3 & 0.89 (0.81 to 0.98$)$ & 0.014 \\
\hline M only & 1 & $0.99(0.82$ to 1.19$)$ & 0.916 & 0 & - & - & 0 & - & - & 0 & - & - \\
\hline \multicolumn{13}{|c|}{ Fractions of distribution } \\
\hline Thirds & 1 & $0.91(0.71$ to 1.16$)$ & 0.451 & 1 & $0.94(0.84$ to 1.05$)$ & 0.277 & 1 & $0.90(0.80$ to 1.01$)$ & 0.076 & 1 & $0.84(0.65$ to 1.08$)$ & 0.170 \\
\hline Quarters & 3 & $0.92(0.81$ to 1.04$)$ & 0.193 & 3 & 0.77 (0.60 to 0.98$)$ & 0.032 & 2 & $0.97(0.75$ to 1.25$)$ & 0.827 & 3 & 0.80 (0.69 to 0.93$)$ & 0.004 \\
\hline Fifths & 7 & 0.94 (0.87 to 1.02$)$ & 0.144 & 5 & 0.96 (0.84 to 1.09$)$ & 0.499 & 6 & $0.94(0.82$ to 1.08$)$ & 0.385 & 3 & 0.89 (0.78 to 1.01$)$ & 0.062 \\
\hline \multicolumn{13}{|c|}{ No of participants } \\
\hline$\geq 50000$ & 3 & 0.91 (0.84 to 0.99$)$ & 0.032 & 2 & 0.83 (0.52 to 1.33$)$ & 0.448 & 1 & $1.01(0.91$ to 1.13$)$ & 0.858 & 2 & 0.86 (0.76 to 0.98$)$ & 0.024 \\
\hline$<50000$ & 8 & 0.95 (0.87 to 1.04$)$ & 0.237 & 7 & 0.93 (0.84 to 1.02$)$ & 0.109 & 8 & 0.92 (0.83 to 1.03$)$ & 0.146 & 5 & 0.86 (0.77 to 0.97$)$ & 0.010 \\
\hline \multicolumn{13}{|l|}{ No of cases } \\
\hline$\geq 1000$ & 5 & 0.95 (0.88 to 1.03$)$ & 0.233 & 4 & $1.01(0.94$ to 1.08$)$ & 0.810 & 6 & 0.96 (0.86 to 1.07$)$ & 0.456 & 3 & 0.91 (0.84 to 0.98$)$ & 0.018 \\
\hline$<1000$ & 6 & $0.91(0.82$ to 1.00$)$ & 0.042 & 5 & 0.75 (0.66 to 0.85$)$ & 0.000 & 3 & $0.87(0.73$ to 1.04$)$ & 0.119 & 4 & 0.78 (0.68 to 0.89$)$ & 0.000 \\
\hline
\end{tabular}


assessment of absolute intake, which they tended to overestimate. ${ }^{58} 59$ However, two studies collected data via a single $24 \mathrm{~h}$ recall and dietary history interviews, respectively. ${ }^{15}{ }^{45}$ These measurements may underestimate true associations between fruit, vegetables, or fruit and vegetables combined consumption and risk of T2D. In addition, calculations of daily consumption differed (such as servings per week, servings per day or grams per day). Although we standardised primary data using a standard portion size of $106 \mathrm{~g}$, conclusions should be interpreted with caution. Another possible explanation for the differences between the studies might be the classification of food groups. Among the studies, GLVs' criteria was inconsistent: three studies included spinach and lettuce; one included spinach and greens; others did not provide any specific description. If they were included with a uniform definition for each group, the associations might differ.

\section{Strengths and limitations}

Compared with previous meta-analyses, ${ }^{5} 89$ our study has several strengths. To the best of our knowledge, this is the largest systematic review and meta-analysis on the intake of fruit and vegetables and risk of T2D. In addition, to examine the shape of these possible associations, we investigated a dose-response relation between fruit, vegetables, or fruit and vegetables combined consumption and risk of T2D. Therefore, the results should be more reliable.

In interpreting the results, several limitations of this meta-analysis should also be acknowledged. First, although in the multivariable analysis we considered a multitude of lifestyle and dietary factors, the possibility of residual confounding or confounding by unmeasured factors, which cannot be ruled out in any observational study, must be acknowledged. Second, we cannot exclude the possibility of recall bias in the assessments of diet based on the FFQs. However, the prospective study design and exclusion of participants with chronic diseases at baseline should minimise such bias. Third, the noticeable limitation of our study was the potential for bias due to the inevitable measurement error, especially for an individual with lower consumption levels. We attempted to reduce measurement error by adjusting for energy intake and using cumulatively averaged intake levels. Fourth, since we had no source of information other than the questionnaire for the identification of T2D, we might have underestimated the incidence of T2D. In addition, subclinical diseases at baseline might have distorted our risk estimate to some extent. Finally, the possible limitation is due to language bias. We attempted to minimise this bias by searching major electronic databases with no language restriction. However, several articles published in non-English languages might not appear in international journal databases, and could be omitted by our searches. ${ }^{60}$

\section{CONCLUSIONS}

In summary, our meta-analysis suggests that higher fruit or GLV intake is associated with a significantly reduced risk of T2D. In addition, the dose-response relations also indicate that relatively high fruit or GLV may still decrease the risk of T2D. Further evidence from preferably randomised controlled studies should explore what kind of fruit or GLV can reduce the risk of T2D.

Contributors ML and ZT conceived and designed the study. ML and YF searched the databases and checked them according to the eligible inclusion and exclusion criteria. ZT helped develop search strategies. XZ and WH extracted quantitative data. YF, XZ and WH analysed the data. ML wrote the draft of the paper. All authors contributed to the writing, review or revision of the paper. ZT is the guarantor.

Funding This research received no specific grant from any funding agency in the public, commercial or not-for-profit sectors.

\section{Competing interests None.}

Provenance and peer review Not commissioned; externally peer reviewed.

Data sharing statement No additional data are available.

Open Access This is an Open Access article distributed in accordance with the Creative Commons Attribution Non Commercial (CC BY-NC 4.0) license, which permits others to distribute, remix, adapt, build upon this work noncommercially, and license their derivative works on different terms, provided the original work is properly cited and the use is non-commercial. See: http:// creativecommons.org/licenses/by-nc/4.0/

\section{REFERENCES}

1. Shaw JE, Sicree RA, Zimmet PZ. Global estimates of the prevalence of diabetes for 2010 and 2030. Diabetes Res Clin Pract 2010;87:4-14.

2. Jonsson B. Revealing the cost of type II diabetes in Europe. Diabetologia 2002;45:S5-12.

3. Zhang $\mathrm{P}$, Zhang X, Brown J, et al. Global healthcare expenditure on diabetes for 2010 and 2030. Diabetes Res Clin Pract 2010;87:293-301.

4. Gillies CL, Abrams KR, Lambert PC, et al. Pharmacological and lifestyle interventions to prevent or delay type 2 diabetes in people with impaired glucose tolerance: systematic review and meta-analysis. BMJ 2007;334:299.

5. Cooper AJ, Forouhi NG, Ye Z, et al. Fruit and vegetable intake and type 2 diabetes: EPIC-InterAct prospective study and meta-analysis. Eur J Clin Nutr 2012;66:1082-92.

6. World Health Organization/UN Food and Agriculture Organization. Diet, nutrition and the prevention of chronic diseases: Report of a Joint FAO/WHO Expert Consultation. Geneva: World Health Org. 2003. (Tech. Rep. Ser., no. 916).

7. Kurotani K, Nanri A, Goto A, et al. Vegetable and fruit intake and risk of type 2 diabetes: Japan Public Health Center-based Prospective Study. Br J Nutr 2013;109:709-17.

8. Hamer M, Chida Y. Intake of fruit, vegetables, and antioxidants and risk of type 2 diabetes: systematic review and meta-analysis. $J$ Hypertens 2007;25:2361-9.

9. Carter P, Gray LJ, Troughton J, et al. Fruit and vegetable intake and incidence of type 2 diabetes mellitus: systematic review and meta-analysis. BMJ 2010;341:c4229.

10. Cooper AJ, Sharp SJ, Lentjes MA, et al. A prospective study of the association between quantity and variety of fruit and vegetable intake and incident type 2 diabetes. Diabetes Care 2012;35:1293-300.

11. Muraki I, Imamura F, Manson JE, et al. Fruit consumption and risk of type 2 diabetes: results from three prospective longitudinal cohort studies. BMJ 2013;347:f5001.

12. Stroup DF, Berlin JA, Morton SC, et al. Meta-analysis of observational studies in epidemiology: a proposal for reporting. Meta-analysis Of Observational Studies in Epidemiology (MOOSE) group. JAMA 2000;283:2008-12.

13. Wong WC, Cheung CS, Hart GJ. Development of a quality assessment tool for systematic reviews of observational studies 
(QATSO) of HIV prevalence in men having sex with men and associated risk behaviours. Emerg Themes Epidemiol 2008;5:23.

14. von $\mathrm{EE}$, Altman DG, Egger $\mathrm{M}$, et al. The Strengthening the Reporting of Observational Studies in Epidemiology (STROBE) statement: guidelines for reporting observational studies. Prev Med 2007;45:247-51.

15. Ford ES, Mokdad AH. Fruit and vegetable consumption and diabetes mellitus incidence among U.S. adults. Prev Med 2001;32:33-9.

16. DerSimonian R, Laird N. Meta-analysis in clinical trials. Control Clin Trials 1986;7:177-88.

17. Dauchet L, Amouyel P, Hercberg S, et al. Fruit and vegetable consumption and risk of coronary heart disease: a meta-analysis of cohort studies. J Nutr 2006;136:2588-93.

18. Greenland S, Longnecker MP. Methods for trend estimation from summarized dose-response data, with applications to meta-analysis. Am J Epidemiol 1992;135:1301-9.

19. Orsini N, Bellocco R, Greenland S. Generalized least squares for trend estimation of summarized dose-response data. Stata $J$ 2006;6:40-57.

20. Orsini N, Li R, Wolk A, et al. Meta-analysis for linear and nonlinear dose-response relations: examples, an evaluation of approximations, and software. Am J Epidemiol 2012;175:66-73.

21. Harrell FE Jr, Lee KL, Pollock BG. Regression models in clinical studies: determining relationships between predictors and response. J Natl Cancer Inst 1988:80:1198-202.

22. Higgins JP, Thompson SG, Deeks JJ, et al. Measuring inconsistency in meta-analyses. BMJ 2003;327:557-60.

23. Begg CB, Mazumdar M. Operating characteristics of a rank correlation test for publication bias. Biometrics 1994;50:1088-101.

24. Egger M, Davey SG, Schneider M, et al. Bias in meta-analysis detected by a simple, graphical test. BMJ 1997;315:629-34.

25. Peters JL, Sutton AJ, Jones DR, et al. Contour-enhanced meta-analysis funnel plots help distinguish publication bias from other causes of asymmetry. J Clin Epidemiol 2008;61:991-6.

26. Bauer F, Beulens JW, van der A DL, et al. Dietary patterns and the risk of type 2 diabetes in overweight and obese individuals. Eur $J$ Nutr 2013;52:1127-34.

27. Eshak ES, Iso H, Mizoue T, et al. Soft drink, $100 \%$ fruit juice, and vegetable juice intakes and risk of diabetes mellitus. Clin Nutr 2013;32:300-8.

28. von RA, Feller S, Bergmann MM, et al. Diet and risk of chronic diseases: results from the first 8 years of follow-up in the EPIC-Potsdam study. Eur J Clin Nutr 2013;67:412-19.

29. Yu R, Woo J, Chan R, et al. Relationship between dietary intake and the development of type 2 diabetes in a Chinese population: the Hong Kong Dietary Survey. Public Health Nutr 2011;14:1133-41.

30. Morimoto A, Ohno Y, Tatsumi Y, et al. Effects of healthy dietary pattern and other lifestyle factors on incidence of diabetes in a rural Japanese population. Asia Pac J Clin Nutr 2012;21:601-8.

31. Martinez-Gonzalez MA, de la Fuente-Arrillaga C, Benito S, et al. Low consumption of fruit and vegetables and risk of chronic disease: a review of the epidemiological evidence and temporal trends among Spanish graduates. Public Health Nutr 2011;14:2309-15.

32. Esposito K, Kastorini CM, Panagiotakos DB, et al. Prevention of type 2 diabetes by dietary patterns: a systematic review of prospective studies and meta-analysis. Metab Syndr Relat Disord 2010;8:471-6.

33. Lodefalk M, Aman J. Food habits, energy and nutrient intake in adolescents with type 1 diabetes mellitus. Diabet Med 2006;23:1225-32.

34. Zhu Y, Zhang Y, Ling W, et al. Fruit consumption is associated with lower carotid intima-media thickness and C-reactive protein levels in patients with type 2 diabetes mellitus. J Am Diet Assoc 2011;111:1536-42.

35. Hosseinpour-Niazi S, Mirmiran P, Sohrab G, et al. Inverse association between fruit, legume, and cereal fiber and the risk of metabolic syndrome: Tehran Lipid and Glucose Study. Diabetes Res Clin Pract 2011:94:276-83.

36. Odegaard AO, Koh WP, Arakawa K, et al. Soft drink and juice consumption and risk of physician-diagnosed incident type 2 diabetes: the Singapore Chinese Health Study. Am J Epidemiol 2010;171:701-8.
37. Hodge AM, English DR, O'Dea K, et al. Dietary patterns and diabetes incidence in the Melbourne Collaborative Cohort Study. Am J Epidemiol 2007;165:603-10.

38. Cai H, Shu XO, Gao YT, et al. A prospective study of dietary patterns and mortality in Chinese women. Epidemiology 2007;18:393-401

39. Sartorelli DS, Cardoso MA. [Association between dietary carbohydrates and type 2 diabetes mellitus: epidemiological evidence]. Arq Bras Endocrinol Metabol 2006:50:415-26.

40. Erber E, Hopping BN, Grandinetti A, et al. Dietary patterns and risk for diabetes: the multiethnic cohort. Diabetes Care 2010;33:532-8.

41. Sluijs I, Beulens JW, van der A DL, et al. Dietary intake of total, animal, and vegetable protein and risk of type 2 diabetes in the European Prospective Investigation into Cancer and Nutrition (EPIC)-NL study. Diabetes Care 2010;33:43-8.

42. Liese $A D$, Weis KE, Schulz M, et al. Food intake patterns associated with incident type 2 diabetes: the Insulin Resistance Atherosclerosis Study. Diabetes Care 2009;32:263-8.

43. Meyer KA, Kushi LH, Jacobs DR Jr, et al. Carbohydrates, dietary fiber, and incident type 2 diabetes in older women. Am J Clin Nutr 2000;71:921-30.

44. Liu S, Serdula M, Janket SJ, et al. A prospective study of fruit and vegetable intake and the risk of type 2 diabetes in women. Diabetes Care 2004;27:2993-6.

45. Montonen J, Jarvinen R, Heliovaara M, et al. Food consumption and the incidence of type II diabetes mellitus. Eur J Clin Nutr 2005;59:441-8.

46. Villegas R, Shu XO, Gao YT, et al. Vegetable but not fruit consumption reduces the risk of type 2 diabetes in Chinese women. J Nutr 2008;138:574-80.

47. Bazzano LA, Li TY, Joshipura KJ, et al. Intake of fruit, vegetables, and fruit juices and risk of diabetes in women. Diabetes Care 2008;31:1311-17.

48. McKee LH, Latner TA. Underutilized sources of dietary fiber: a review. Plant Foods Hum Nutr 2000;55:285-304.

49. Liese AD, Roach AK, Sparks KC, et al. Whole-grain intake and insulin sensitivity: the Insulin Resistance Atherosclerosis Study. Am $J$ Clin Nutr 2003;78:965-71.

50. Schulze MB, Schulz M, Heidemann C, et al. Fiber and magnesium intake and incidence of type 2 diabetes: a prospective study and meta-analysis. Arch Intern Med 2007;167:956-65.

51. Jenkins DJ, Wolever TM, Buckley G, et al. Low-glycemic-index starchy foods in the diabetic diet. Am J Clin Nutr 1988;48:248-54.

52. Tarwadi K, Agte V. Potential of commonly consumed green leafy vegetables for their antioxidant capacity and its linkage with the micronutrient profile. Int J Food Sci Nutr 2003;54:417-25.

53. Hozawa A, Jacobs DR Jr, Steffes MW, et al. Associations of serum carotenoid concentrations with the development of diabetes and with insulin concentration: interaction with smoking: the Coronary Artery Risk Development in Young Adults (CARDIA) Study. Am J Epidemiol 2006;163:929-37.

54. Harding $\mathrm{AH}$, Wareham NJ, Bingham SA, et al. Plasma vitamin $\mathrm{C}$ level, fruit and vegetable consumption, and the risk of new-onset type 2 diabetes mellitus: the European prospective investigation of cancer -Norfolk prospective study. Arch Intern Med 2008;168:1493-9.

55. Liu S, Ajani U, Chae C, et al. Long-term beta-carotene supplementation and risk of type 2 diabetes mellitus: a randomized controlled trial. JAMA 1999;282:1073-5.

56. Ceriello A, Motz E. Is oxidative stress the pathogenic mechanism underlying insulin resistance, diabetes, and cardiovascular disease? The common soil hypothesis revisited. Arterioscler Thromb Vasc Biol 2004;24:816-23.

57. Larsson SC, Wolk A. Magnesium intake and risk of type 2 diabetes: a meta-analysis. J Intern Med 2007;262:208-14.

58. Feskanich D, Rimm EB, Giovannucci EL, et al. Reproducibility and validity of food intake measurements from a semiquantitative food frequency questionnaire. J Am Diet Assoc 1993;93:790-6.

59. Bingham SA, Welch AA, McTaggart A, et al. Nutritional methods in the European Prospective Investigation of Cancer in Norfolk. Public Health Nutr 2001;4:847-58.

60. Li M, Hou W, Zhang X, et al. Hyperuricemia and risk of stroke: a systematic review and meta-analysis of prospective studies. Atherosclerosis 2014;232:265-70. 